\title{
ASTROMETRY AND GALACTIC STRUCTURE
}

\author{
S.R. MAJEWSKI \\ The Observatories of the Carnegie Institution of Washington \\ 813 Santa Barbara Street \\ Pasadena, CA 91101 \\ U.S.A.
}

\begin{abstract}
The application of astrometric surveys to understanding the structure and kinematics of the Milky Way is discussed, with particular emphasis on some of the issues expected to be addressed in the next decade or so. It is shown that the results of complete proper motion surveys of field stars at the Galactic poles are in remarkable agreement concerning the global kinematics of stars as a function of distance, but that significant differences exist in how these data are decomposed to derive the kinematics of individual Galactic components. The status and future prospects regarding the measurement of proper motions and orbits for Galactic globular clusters and satellites are also discussed.
\end{abstract}

\section{Introduction}

Astrometry has long benefitted from wide-field imaging, and remains one endeavor that still relies heavily on the photographic plate. While some pioneering work is being conducted on the application of CCDs toward trigonometric parallaxes (see contributions to this conference by Monet and Tinney), to my knowledge, no study has yet applied CCD data to the study of proper motions, nor successfully attempted to combine CCD and photographic data for this purpose, although this hand is now forced. Perhaps no other field of astronomy will be as significantly impacted in the near term by the unfortunate decision by Kodak to discontinue production of photographic glass plates, which, because of their stability and large area, have been the proven mainstay for long baseline proper motion analyses.

This review will concentrate on the application of proper motion data to the study of Galactic structure and kinematics. A number of large astrometric projects are just now getting underway, often with only initial results having been reported. Rather than focussing entirely on a comprehensive review of past work, I would like instead to include some preview of the important astrometric results we can expect in the next decade or so in the study of field stars, globular clusters, and satellite galaxies of the Milky Way.

\section{Deep, Complete Field Star Surveys at the Galactic Poles}

Earlier this century, Kapteyn (1906) set in motion an unprecedented organizational effort to bring together the labors of astronomers worldwide to address a single problem, that of 'the structure 
of the sidereal world'. The plan called for the assemblage of a Durchmusterung of magnitudes, spectral classes or colors, proper motions, radial velocities, and parallaxes for complete samples of stars in 206 Selected Areas to the limits accessible and with what was viewed at the time as a reasonable allocation of the net available resources of astronomy. Viewed from the modern context, the commitment of (what was then considered large) telescope time ('guaranteed time observing'), personnel, and other resources by a large number of observatory directors toward this singular effort to obtain complete surveys of star counts, colors and astrometry is nothing short of remarkable (cf. the Appendix of Kapteyn 1906; Kapteyn 1911; van Rhijn 1923).

An advantage of Kapteyn's approach in compiling complete surveys of stars is that it is free of selection effects which may bias the measurement of specific Galactic parameters or correlations. This advantage is more fully appreciated in the post-Eggen, Lynden-Bell \& Sandage (1962) era where the issue of selection bias is an ever increasing concern with regard to the interpretation of Galactic structure surveys. For example, halo star samples have often been compiled from solar neighborhood stars on the basis of large proper motions or low abundances, but it is now questionable whether samples defined in these ways a) yield complete and representative samples of halo stars, or b) allow admixtures with other populations, due to overlap of kinematical and abundance properties. In either of these cases, the measurement of any parameter that is correlated to the selection parameter is susceptible to the inherent selection biases.

An increasingly popular strategy (though not necessarily a more straightforward one), with roots in Kapteyn's approach but now probing to deeper $(\mathrm{V}>16)$ magnitudes, is to compile complete samples of stars and try to discern the best 'mixture model' (cf. Nemec \& Nemec 1991) for the stellar populations across the entire range of the observed parameter space. With considerably less access to large telescopes than Kapteyn was able to commandeer, deep, complete stellar surveys that include proper motions have most often concentrated in two regions, the South and North Galactic Poles (which, in Kapteyn's system, are represented by Selected Areas 141 and 57, respectively), though some work has been done in a few other areas (Chiu 1980; see also Bienayme et al., Ojha et al. and Kharchenko et al. in these proceedings). Some deep surveys are in progress that will cover large portions of the sky (cf. Schilbach et al., this conference; Table 1 of the contribution by van Altena summarizes the various Schmidt plate digitization efforts, some which will include proper motions as an end product). It is also worth pointing out the important contribution that will be made by the now completed, but yet unpublished, Lick Northern Proper Motion Survey (NPM; cf. Klemola et al. 1987) with respect to galaxies, and its counterpart, currently in progress, the Yale Southern Proper Motion Survey (SPM; cf. van Altena et al. 1990). The NPM and SPM are not complete surveys, but are unbiased in the selection of candidate stars in each survey area.

The advantages of observing directly at a Galactic pole are low reddening, easiest access (in terms of magnitudes) to in situ populations of thick disk and halo stars, and the fact that proper motions transform directly into $u$ and $v$ velocities, motions with respect to the Local Standard of Rest (LSR) in the directions of the Galactic anticenter and rotation, respectively. Table 1 summarizes the deep proper motion surveys that have been conducted in these two directions of the sky (along with the NPM survey analysis by Hanson 1989), and the discussion is further limited to these particular surveys of field stars. In general, two approaches are adopted, as dictated by the desire to obtain absolute proper motions with respect to the extragalactic reference frame: 1) shallow surveys with large-area Schmidt plates that can harvest an appreciable number of the low density of bright galaxies, or 2) smaller area surveys which, however, go deep to 
uncover large numbers of fainter galaxies.

Table 1. Unbiased proper motion surveys at the Galactic poles

\begin{tabular}{|c|c|c|c|c|c|c|c|}
\hline Survey & Region & Plates & $\begin{array}{c}\Delta \tau \\
\text { (years) }\end{array}$ & $\begin{array}{c}\text { area } \\
\left(\mathrm{deg}^{2}\right)\end{array}$ & $\mathbf{N}_{\text {atars }}$ & $\mathbf{m}_{\text {ymat }}$ & $\begin{array}{c}\varepsilon_{\mu}\left(m_{\text {vimtit }}-2\right) \\
\text { ("/cent) }\end{array}$ \\
\hline Murray (1986) & SGP & UK Schmidt & 6 & 20 & $5500^{\star}$ & $\begin{array}{l}B=17.5 \\
V=17.0\end{array}$ & 0.7 \\
\hline Hanson (1989) & NPM & Lick Astrograph & 27 & 22200 & 60000 & $15<\mathrm{m}_{\mathrm{pg}}<17$ & 0.5 \\
\hline Spaenhauer (1989) & NGP & Lick Astrograph & 37 & 2.6 & 1200 & B 18.0 & 0.3 \\
\hline $\begin{array}{l}\text { Kharchenko et al. } \\
\text { (this conference) }\end{array}$ & NGP & $\begin{array}{c}\text { Tautenburg } \\
\text { Schmidt }\end{array}$ & $20-25$ & $\begin{array}{c}8.9+ \\
7.5\end{array}$ & $\sim 10000^{\circ}$ & $\begin{array}{l}B=19 \& \\
B=20.5\end{array}$ & 0.3 \\
\hline Soubiran (1993) & NGP & $\begin{array}{l}\text { 48", Tautenburg, } \\
\text { OCA Schmidts }\end{array}$ & 40 & 7 & 2370 & $\mathrm{~V} \sim 18$ & 0.2 \\
\hline Lattanzi et al. (1993) & ) NGP & 48" Schmidt & 40 & 45 & $\sim 36000$ & $V=18$ & 0.4 \\
\hline Reid (1990) & NGP & 48" Schmidt & 21 & 28 & 35000 & V 20 & 1.0 \\
\hline Chiu (1980) & NGP & 200", KPNO 4m & 25 & 0.1 & 175 & $V=21$ & 0.2 \\
\hline Majewski (1992) & NGP & KPNO 4m & 16 & 0.3 & 900 & $B=22.5$ & 0.08 \\
\hline Guo et al. (1993ab) & SGP & CTIO 4m & 11 & 0.5 & $5000^{2}$ & $B=22.5$ & $0.1-0.2$ \\
\hline
\end{tabular}

- The survey field contains the globular cluster NGC 288 (SGP) or M 3 (NGP), which contribute to the number of stars.

Figure 1 illustrates the derived global velocity components $v, \sigma_{\mu}$ and $\sigma_{v}$ for complete samples of stars as a function of $|z|$, the distance from the Galactic plane, for several of the Table 1 surveys (the Guo et al., Lattanzi et al., and Kharchenko et al. studies are still in progress, while the Chiu data have been left out because of the systematic errors identified in Majewski 1992). It can be seen that the various polar surveys give fairly good agreement for $v(z), \sigma_{u}(z)$, and $\sigma_{v}(z)$ over those distances of overlap. From their preliminary analysis, Guo et al. (1993b) also report rough consistency with the $\sigma_{\mu}$ and $\sigma_{v}$ results of Murray (1986) and Majewski (1992), and a similar $v$ gradient $\left(-35 \pm 2 \mathrm{~km} / \mathrm{s}^{-1} \mathrm{kpc}^{-1}\right)$ to the other studies for $|\mathrm{z}|<1.5 \mathrm{kpc}$. This general agreement among the various surveys is especially gratifying given the variety of strategies taken to obtain the proper motions and distances for individual stars within each survey. However, in spite of the reasonably good agreement in the global kinematic trends among the complete surveys in Fig. 1, the derived kinematical properties for specific Galactic components range broadly among the Table 1 surveys. For example, Soubiran (1993, and this conference) finds a $v_{\text {rot }}$ for the thick disk of $179 \pm 16 \mathrm{~km} / \mathrm{s}^{-1}$, Spaenhauer (1989) finds $v_{\mathrm{rot}}=140-160 \mathrm{~km} / \mathrm{s}^{-1}$, while Majewski (1992) claims that the thick disk rotational velocity is not single-valued, but that it exhibits a gradient in rotational velocity from only a modest lag behind the LSR at low $z$ to a low rotational velocity of $\sim 100 \mathrm{~km} / \mathrm{s}^{-1}$ at $z \sim 5.5 \mathrm{kpc}$. The general accord seen in Fig. 1 suggests, therefore, that some disagreements in the derived properties of the Galactic stellar populations come principally from differences in interpreting the distribution of the stars into these populations. Moreover, the even broader distribution in kinematic properties of the various stellar populations from tracer and proper motion or metallicity-selected surveys (see for example Fig. 6 of Majewski 1993) may derive from compounding the uncertainty in the decomposition of Galactic stellar populations with the effects of selection bias.

How is the transition from thin disk to thick (extended, Intermediate Population II) disk to 

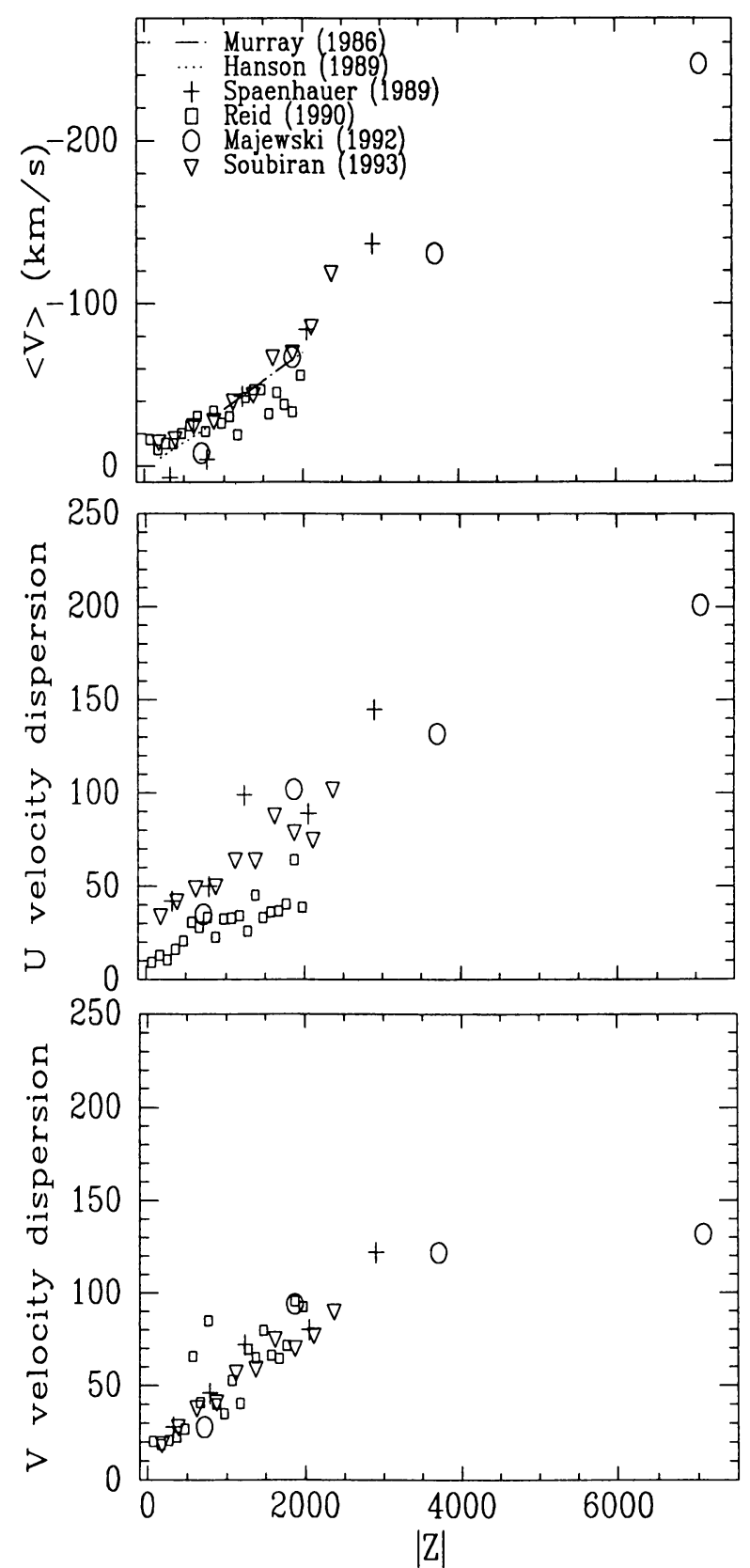

Figure 1. The run of $v, \sigma_{k}$ and $\sigma_{v}$ with distance from the Galactic plane, $|z|$ as derived from complete surveys of proper motions at the Galactic poles, referenced in Table 1. Error bars have been left out for clarity, but generally range from a few $\mathrm{km} / \mathrm{s}^{-1}$ to $\sim 20 \mathrm{~km} / \mathrm{s}^{-1}$ vertically. 
halo manifested in Fig. 1? Without a priori knowledge of the mixing ratios of the populations at each distance, a number of scenarios might be possible, with any combination of mean kinematic properties between populations and possibly more complicated spatial dependencies within each population. Much could be gained if one could identify distance intervals where specific Galactic populations dominate, attribute the trends seen over that distance interval to that single component, and then extrapolate those trends in order to understand regions of overlap. One approach might be to use density laws as derived from starcount analyses. The starcount problem is, however, not yet resolved unambiguously. As an example of the state of this work, note the widely disparate thick disk parameters (scaleheight, local density relative to thin disk) derived in the contributions to this conference by Robin et al. (800 pc, $4.5 \%)$ and by Reid \& Majewski (1400 pc, $2.5 \%)$. With such uncertainty in the spatial distribution of the thick disk, it is no wonder that different groups that find similar global kinematic properties can decompose these kinematics into widely different properties for individual Galactic components. For comparison, the thick disk population as described in the kinematic studies by Spaenhauer, Majewski and by Soubiran have density parameters (scaleheight, local density relative to thin disk) of (1300 pc, $2 \%),(1400 \mathrm{pc}, 3.8 \%)$ and $(700 \mathrm{pc}, 6 \%)$, respectively, while Guo et al. (1993) derive a scaleheight of $1715 \pm 256 \mathrm{pc}$. Ultimately, of course, the decomposition of the Galaxy into components must satisfy both kinematic and starcount constraints simultaneously. Such multivariate analyses are already being developed and utilized (Robin \& Créze 1986; Ratnatunga et al. 1989; Casertano et al. 1990; Nemec \& Nemec 1991; Bienayme et al., this conference).

While the spatial extent, and therefore the kinematics, of the thick disk are perhaps still quite uncertain, if we can identify some $|z|$ beyond which the thick disk does not contribute significantly and for which the halo is the dominant component then some surveys in Table 1 should be able to derive the transverse kinematical components of the halo from relatively pure, in situ samples. As shown by Majewski (1993), a 'safe', conservative $z$ distance that accounts for the most extensive thick disks that have been suggested (1750 pc scaleheight, $4 \%$ local normalization) would be $13 \mathrm{kpc}$ or more in order to have less than $20 \%$ thick disk contamination. This distance is fairly prohibitive for the magnitude ranges of the Table 1 surveys (unless giant or subgiant stars could be identified within these samples). A more liberal distance might be something like $z=5 \mathrm{kpc}$, but it should be kept in mind that even at this distance we would expect $40 \%$ of stars to be from the thick disk for the density laws of the type quoted above by Reid \& Majewski and Spaenhauer. In his decomposition into thick disk and halo, Majewski (1992) found that most of his halo stars lay beyond $5 \mathrm{kpc}$, and for these he obtained the surprising result that they seemed to be in net retrograde rotation, with $\left\langle v_{\text {ro }}\right\rangle=-47 \pm 9 \mathrm{~km} / \mathrm{s}^{-1}$. If true, this result would be an important constraint on formation models of the halo, strongly favouring accretion or Searle \& Zinn (1978) type models over simple collapse models. Reid's (1990) survey also reached these distances, and he also obtained a net retrograde rotation for $\sim 200$ dwarf stars at $\langle z>\sim 13 \mathrm{kpc}$, albeit with large enough error bars as to be consistent with nonrotation, $\left\langle v_{\text {ror }}\right\rangle=-20 \pm 30 \mathrm{~km} / \mathrm{s}^{-1}$. The SGP survey of Guo et al. (1993b) is most similar to Majewski's, but in their preliminary analysis they find a slightly prograde halo for $z>5 \mathbf{k p c}^{\dagger}$. Clearly more work must be devoted to resolving this discrepancy between the various Table 1 surveys, especially given the evolutionary implications of a retrograde halo, but other alluring

\footnotetext{
' One possible contribution to the difference could be that Majewski (1992) attempted to decompose the $5<z<10 \mathrm{kpc}$ stars into thick disk $(\sim 20 \%)$ and halo components $(\sim 80 \%)$ while the Guo et al. result apparently applies to all stars.
} 
evidence is given by two surveys using selected samples of solar neighborhood stars. Allen et al. (1991) have calculated orbits for the $[\mathrm{Fe} / \mathrm{H}]<-2.0$ stars in Schuster \& Nissen (1988); they find a significant feature in the distribution of angular momentum such that stars having a $z_{\max }$ (the maximum distance from the Galactic plane) less than $4 \mathrm{kpc}$ have a mean $v_{\mathrm{rot}}=50 \mathrm{~km} / \mathrm{s}^{-1}$ while 10 of 13 stars in their sample with $z_{\max }>4 \mathrm{kpc}$ have no or a retrograde velocity, and yield a mean $v_{\mathrm{rot}}$ of $-87 \mathrm{~km} / \mathrm{s}^{-1}$. Carney et al. (private communication) have calculated improved orbital elements for their proper motion-selected sample (cf. Carney et al. 1990) and for their 33 stars with $z_{\max }>5 \mathrm{kpc}$ they obtain $\langle v\rangle=-270 \pm 21 \mathrm{~km} / \mathrm{s}^{-1}$, in almost perfect agreement with Majewski's (1992) result ${ }^{\dagger \dagger}$.

\section{Astrometry of Globular Clusters and Galactic Satellites}

The next decade should see new forays into our understanding of globular clusters and Galactic satellites with an expected wealth of new astrometric results for these objects. Full space velocities allow orbits to be calculated and bring a new dimension to studies of cluster/satellite origins, destruction and relations to stellar populations and Galactic structure, as well as enable a more precise dynamical mapping of the Galactic gravitational potential. Previously, these analyses could rely only on radial velocities and used statistical arguments to account for incomplete orbital data. Some of the questions for which we may expect advances in the next decade or so of research in this area are:

1) what is the appropriate distinction between disk and halo clusters?

2) is the globular cluster gap at $40<R<70 \mathrm{kpc}$ due to a chance positioning of the cluster ensemble or is it due to specific evolutionary processes?

3) have some clusters been torn from the Magellanic Clouds or other satellite galaxies (cf. Lin \& Richer 1992)?

4) how do the orbits of clusters relate to their other properties (mass, tidal radius, shape, concentration) and what does this tell us about processes of cluster destruction?

5) is there a relationship between the clusters and the field stars evidenced in their kinematics?

6) do the claims (Rodgers \& Paltoglou 1984; van den Bergh 1993) of a retrograde subpopulation of halo clusters, at metallicities $-1.3 \geq[\mathrm{Fe} / \mathrm{H}] \geq-1.7$, hold up when three-dimensional space velocities are included in the analysis? Like the possible retrograde halo field star population described above, this retrograde cluster subpopulation has been taken as circumstantial evidence for accretion in the Milky Way's past;

7) how do the orbits of halo clusters relate to Zinn's (1993) division into young and old groups based on horizontal branch morphology? Zinn has suggested that the oblate configuration of old halo clusters, with a net rotational velocity (based on radial velocities alone) of $70 \pm 22 \mathrm{~km} / \mathrm{s}^{-1}$, a radial velocity dispersion of $89 \pm 9 \mathrm{~km} / \mathrm{s}^{-1}$, and a metallicity gradient from $R=6$ to $40 \mathrm{kpc}$, may be related to, and smoothly join to, that of the disk globular cluster system. The more spherical distribution of young halo clusters, with a net (retrograde) rotational velocity of $-64 \pm 74 \mathrm{~km} / \mathrm{s}^{-1}$, a velocity dispersion of $149 \pm 24 \mathrm{~km} / \mathrm{s}^{-1}$, and no discernible metallicity gradient, may have been accreted;

8) Kunkel \& Demers (1976) and Lynden-Bell (1982) have suggested that the satellites of the

\footnotetext{
tt While this result is without correction for unselected stars with small proper motion, this correction should be small for stars near the sun required to have $z_{\max }>5 \mathrm{kpc}$.
} 
Milky Way are not randomly distributed, but rather aligned in one or more great planes in the sky, perhaps as a result of accretion of larger bodies. The radial velocities of satellites and some of the outer halo globulars (all of which are Zinn 'young halo' clusters, cf. Majewski 1994) suggest Keplerian motion in these planes (Kunkel 1979), but full space velocities are required to check whether these satellites and clusters share a common direction of angular momentum;

9) are the orbits of the distant satellites and clusters radial or isotropic? Derived dynamical masses of the Galaxy vary by as much as a factor of four at these extremes of orbital properties.

Globular cluster proper motions are only now beginning to be obtained with reliability. An important contribution is that recently by Cudworth \& Hanson (1993), which combined proper motion measurements of $\mathbf{1 4}$ globular clusters with respect to field stars and an analysis of the mean field star motions expected on the basis of the NPM survey with respect to galaxies. However, it is good to remember the inherent difficulties of using field stars as absolute proper motion references, with an important dependence on the adopted solar motion and/or models of field star secular parallaxes (cf. Cudworth 1993; Majewski 1993). Although a variety of schemes have been tried to overcome these problems (cf. Brosche et al. 1991; Cudworth 1993), the most reliable method is to measure proper motions directly with respect to galaxies or QSOs, a technique just now beginning to be exploited. As with the field stars surveys, two approaches are being taken in this enterprise, with the goal to obtain as many compact galaxies or QSOs as possible to reduce systematic and random errors:

1) Schmidt plate astrometry over large areas to reach a sufficient number of bright galaxies which have low angular density (cf. Scholz et al. and Odenkirchen et al. at this conference), and

2) very deep astrometry in small areas with large, long focal length telescopes to reach the higher density of fainter galaxies and QSOs (Guo et al. 1993; Majewski \& Cudworth 1993; Schweitzer et al. 1993; Cudworth et al. 1993).

Table 2 summarizes most of the space velocities presently known for globular clusters (but does not include the results for M 3, M 92 and M 2 by Scholz et al. [1993], and by Odenkirchen et al. and Geffert et al. at this conference). From the velocity data, Luis Aguilar (UNAM) has calculated orbits within a Bahcall et al. (1983) Galactic potential. Some of the properties of these calculated orbits - namely, the mean peri- and apogalactica, the mean eccentricities $e=\left(R_{\text {apo }}-R_{\text {per }}\right) /\left(R_{\text {apo }}+R_{\text {per }}\right)$, and $z_{\max }-$ are provided in Table 2 . While the total number of clusters with known space velocities is still meager, and while the calculated parameters in Table 2 are somewhat preliminary, several interesting features of the Table 2 globulars can already be discerned. The first is the rather blurry distinction between disk and (old) halo globular clusters. Earlier, Zinn (1985) had made the distinction between these populations at $[\mathrm{Fe} / \mathrm{H}]=-0.8$, but note the number of globular clusters classified as 'halo' under this definition that have lower $z_{\max }$ than the classical disk globular cluster 47 Tuc. This includes such metal-poor clusters as $M 22$ $([\mathrm{Fe} / \mathrm{H}]=-1.75)$ and $\mathrm{N} 6397([\mathrm{Fe} / \mathrm{H}]=-1.91)$ which have similar $R_{\mathrm{apo}}$ to 47 Tuc. Another way to characterize the orbits is through the parameter $\Psi=\sin ^{-1}\left(z_{\text {max }} / R_{\text {apo }}\right)$, which gives some indication of the maximum possible inclination to the Galactic disk of any cluster. While the old halo clusters span almost the entire range of $\Psi$ - from disk cluster-like to $90^{\circ}$ - the young clusters tend to very high values for this ratio, showing that all the young halo clusters studied to date spend a fraction of their time moving at large inclination angles to the disk. The statistical sample is poor, especially for the young halo clusters, but it is interesting that a larger fraction of young halo clusters in Table 2 are in net retrograde rotation than old halo clusters, and that 
the former tend to have higher mean $z_{\max }$ and $R_{\text {apo }}$. These differences in the orbital properties between young and old halo clusters are consistent with their present spatial distributions in spherical and oblate configurations, respectively. On the other hand, no significant trends appear to exist between the orbital properties of clusters and $[\mathrm{Fe} / \mathrm{H}]$, except that the paradigm disk clusters $\mathrm{M} 71$ and 47 Tuc have both high $[\mathrm{Fe} / \mathrm{H}]$ and very disk-like orbits.

Table 2. Measured and derived orbital parameters for globular clusters

\begin{tabular}{|c|c|c|c|c|c|c|c|c|c|c|c|c|c|c|}
\hline duster & & & & & & & & & $\boldsymbol{R}_{\text {por }}$ & $\boldsymbol{R}_{\text {apo }}$ & $e$ & $\mathbf{Z}_{\max }$ & $\Psi$ & \\
\hline I 71 & D & -0.58 & -2 & (15) & 177 & (12) & -3 & & 4.5 & 6.7 & 0.20 & 0.3 & 3 & \\
\hline 2 & OH & 1.4 & 3 & (5) & 167 & (21) & -40 & (15 & & 2.5 & 0.20 & 0.7 & 16 & \\
\hline & OH & & -55 & (4) & 35 & & 7 & & & & & & & \\
\hline 397 & OH & & 24 & (6) & 126 & (12) & -105 & (1 & & & & .4 & 23 & \\
\hline & OH & o. & 20 & (12) & 144 & (3 & -44 & (26) & & 3.9 & & 2.5 & 40 & \\
\hline 22 & OH & & 167 & (5) & 180 & (1) & -106 & $(2 ?$ & & & & 2.6 & 17 & \\
\hline I 1 & OH & & -38 & (19) & 115 & & 134 & & & & & .5 & & \\
\hline 1 & D & & -19 & (33) & 184 & & 41 & & & & & .5 & & \\
\hline & OH & & 202 & (9) & 55 & (9) & -129 & & & & & .8 & 36 & \\
\hline 1 & OH & 7 & -21 & (34) & 93 & (41) & -45 & $(42$ & 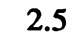 & 10.2 & & 4.9 & 29 & \\
\hline 3 & YH & - & 21 & (42) & -28 & (4 & -82 & (32) & & & & .6 & 69 & \\
\hline 28 & OH & & 6 & (41) & -80 & & 54 & & & & & & 50 & \\
\hline 9 & $\mathrm{OH}$ & -2.2 & 38 & (52) & 4 & & 81 & (37 & & & & 1 & 78 & \\
\hline 13 & $\mathrm{OH}$ & -1.6 & 287 & (54) & -56 & (49) & -116 & (26) & .7 & & & 9.5 & 30 & \\
\hline 2 & YH & -1.66 & 181 & (134) & 237 & (137) & -128 & (24) & & 18.3 & & 14.9 & 55 & \\
\hline al 5 & YH & -1.47 & -118 & (17) & -4 & (30) & 55 & (17) & & & & 6.4 & 76 & \\
\hline & $\mathrm{OH}$ & & -404 & & 21 & & -276 & & & & & 0.8 & 80 & \\
\hline & OH & -1.58 & 85 & (58) & -69 & & -278 & (64) & & & & 22.9 & 78 & \\
\hline 4147 & YH & -1.80 & 151 & (100) & 219 & (98) & 161 & (22) & & & & 24.3 & 71 & \\
\hline al 13 & YH & -1.79 & 173 & (35) & -61 & (39) & -124 & (37) & 20.5 & 26.4 & & 25.8 & 78 & \\
\hline 5466 & $\mathrm{OH}$ & -2.22 & 261 & (98) & -111 & (97) & 221 & (29) & 7.7 & 29.2 & 0.58 & 29.1 & 85 & \\
\hline al $3^{a}$ & $\mathrm{YH}$ & -1.57 & -248 & (95) & 149 & (102) & 203 & (83) & 23. & 218. & 0.81 & 147. & 42 & \\
\hline
\end{tabular}

Notes to Table 2. Cluster classes are as follows: $\mathrm{D}=$ disk, $\mathrm{OH}=\mathrm{Zinn}$ 's (1993) old halo, $\mathrm{YH}=\mathrm{Zinn}$ 's young halo. Velocities in $\mathrm{km} / \mathrm{s}^{-1}$, distances in kpc. References for the derived space velocities are as follows: $B=$ Brosche et al. (1991), C = Cudworth et al. (1993), CH = Cudworth \& Hanson (1993), G = Guo et al. (1993a), $M=$ Majewski \& Cudworth (1993), $S=$ Schweitzer et al. (1993). ' The orbital parameters for Pal 3 are very uncertain due both to the large velocity errors as well as that its orbit goes beyond the computational grid used to compute the 2-D part of the potential and for which only the spherical components of the Galactic potential are considered.

Clearly in-depth exploration of many of the issues outlined above must await the additional cluster motions that will be measured in the next decade. We can also expect orbits to be derived for a number of Galactic satellites, a rich prospect for studies of Galactic evolution. First results have already been obtained for the LMC (Lin 1993) and Ursa Minor and Draco (Scholz \& Irwin, this conference) and are consistent with their orbiting within the Magellanic Stream plane described by Lynden-Bell (1982). That all three satellites have the same direction of angular 
momentum is support for the notion that they may derive from the breakup of a single, larger, progenitor satellite.

\section{Acknowledgements}

I am very grateful to Luis Aguilar for his work to produce the orbital elements presented here. I would also like to thank my collaborator Kyle Cudworth for continuing discussions and comments, and to B. Carney, X. Guo, M. Lattanzi, N. Reid, and R. Scholz for helpful communications and/or unpublished data. This work was supported by Hubble Fellowship Grant Number HF-1036.01-92A, awarded to the Space Telescope Science Institute which is operated by the Association of Universities for Research in Astronomy, Inc. for NASA under Contract No. NAS5-26555.

\section{References}

Allen, C., Schuster, W.J. and Poveda, A., 1991. Astron. Astrophys., 244, 280.

Bahcall, J.N., Schmidt, M. and Soneira, R.M., 1983. Astrophys. J., 265, 730.

Brosche, P., Tucholke, H.-J., Klemola, A.R., Ninkovic, S., Geffert, M. and Doerenkamp, P., 1991. Astron. J., 102, 2022.

Carney, B.W., Aguilar, L., Latham, D.W. and Laird, J.W., 1990. Astron. J., 99, 201.

Casertano, S., Ratnatunga, K.U. and Bahcall, J.N., 1990. Astrophys. J., 357, 435.

Chiu, L.-T., 1980. Astrophys. J. Suppl., 44, 31.

Cudworth, K.M., 1993. In 'Galaxy Evolution: The Milky Way Perspective’, ASP Conf. Ser. 49, ed. S.R. Majewski, ASP San Francisco, p. 141.

Cudworth, K.M. and Hanson, R.B., 1993. Astron. J., 105, 168.

Cudworth, K.M., Takamiya, M., Majewski, S.R. and Peterson, R., 1993. B.A.A.S., 25, 885.

Eggen, O.J., Lynden-Bell, D. and Sandage, A.R., 1962. Astrophys. J., 136, 748.

Guo, X., Girard, T.M., van Altena, W.F. and Lopez, C.E., 1993a. Astron. J., 105, 2182.

Guo, X., Girard, T.M., van Altena, W.F. and Lopez, C.E., 1993b. In 'Galactic and Solar System Optical Astrometry', in press.

Hanson, R.B., 1989. B.A.A.S., 21, 1107.

Kapteyn, J.C., 1906. 'Plan of Selected Areas', Astronomical Laboratory, Groningen.

Kapteyn, J.C., 1911. 'First and Second Reports on the Progress of the Plan of Selected Areas', Astronomical Laboratory, Groningen.

Klemola, A.R., Jones, B.F. and Hanson, R.B., 1987. Astron. J., 94, 501.

Kunkel, W.E.; 1979. Astrophys. J., 228, 718.

Kunkel, W.E. and Demers, S., 1976. R. Greenwich Obs. Bull., 182, 241.

Lattanzi, M.G., Spagna, A., Lasker, B.M., Massone, G., McLean, B., Bucciarelli, B. and Postman, M., 1993. In 'Galactic and Solar System Optical Astrometry', in press.

Lin, D.N.C., 1993. B.A.A.S., 25, 783.

Lin, D.N.C. and Richer, H.B., 1992. Astrophys. J. Lett., 388, L57.

Lynden-Bell, D., 1982. The Observatory, 102, 202.

Majewski, S.R., 1992. Astrophys. J. Suppl., 78, 87.

Majewski, S.R., 1993. ARAA, 31, 575. 
Majewski, S.R., 1994. Astrophys. J. Lett., submitted.

Majewski, S.R. and Cudworth, K.M., 1993. Publ. Astron. Soc. Pacific, 105, 987.

Murray, C.A., 1986. Mon. Not. R. astron. Soc., 223, 649.

Nemec, J. and Nemec, A.F.L., 1991. Publ. Astron. Soc. Pacific, 103, 95.

Reid, I.N., 1990. Mon. Not. R. astron. Soc., 247, 70.

Robin, A. and Créze, M., 1986. Astron. Astrophys. Suppl., 64, 53.

Rodgers, A.W. and Paltoglou, G., 1984. Astrophys. J. Lett., 283, L5.

Scholz, R.-D., Odenkirchen, M. and Irwin, M.J., 1993. Mon. Not. R. astron. Soc., 264, 579.

Schuster, W.J. and Nissen, P.E., 1988. Astron. Astrophys. Suppl., 73, 225.

Schweizer, A., Cudworth, K.M. and Majewski, S.R., 1993. In 'The Globular Cluster - Galaxy

Connection', ASP Conf. Series 48, eds. G. Smith and J. Brodie, ASP San Francisco, p. 113.

Searle, L. and Zinn, R., 1978. Astrophys. J., 225, 357.

Soubiran, C., 1993. Astron. Astrophys., 274, 181.

Spaenhauer, A., 1989. Contrib. Van Vleck Obs., 9, 45.

van Altena, W.F., Girard, T., Lopez, C.E., Lopez, J.A. and Molina, E., 1990. In 'Inertial

Coordinate System on the Sky', IAU Symp. 141, eds. J.H. Lieske and V.K. Abalakin, Kluwer

Academic Publishers, Dordrecht, p. 419.

van den Bergh, S., 1993. Astron. J., 105, 971.

van Rhijn, P.J., 1923. 'Third Report on the Progress of the Plan of Selected Areas', Astronomical Laboratory, Groningen.

Zinn, R., 1985. Astrophys. J., 293, 424.

Zinn, R., 1993. In 'The Globular Cluster - Galaxy Connection', ASP Conf. Ser. 48, eds. G. Smith and J. Brodie, ASP San Francisco, p. 38. 